\title{
Image Quality
}

\author{
Patrick S. Cheatham
}

Pacific-Sierra Research Corp.

1456 Cloverfield Boulevard

Santa Monica, CA 90404

The term image quality can, unfortunately, apply to anything from a public relations firm's discussion to a comparison between corner drugstores' film processing. If we narrow the discussion to optical systems, we clarify the problem somewhat, but only slightly. We are still faced with a multitude of image quality measures-all different, and all couched in different terminology. Optical designers speak of MTF values, digital processors talk about summations of before and after image differences, pattern recognition engineers allude to correlation values, and radar imagers use side-lobe response values measured in decibels. Further complexity is introduced by terms such as information content, bandwidth, Strehl ratios, and, of course, limiting resolution. The problem is to compare these different yardsticks and try to establish some concrete ideas about evaluation of a final image. We need to establish the image attributes which are the most important to perception of the image in question and then begin to apply the different system parameters to those attributes.

This special issue is an attempt to discuss these topics and bring together viewpoints from different fields, allowing some interaction between researchers using different concepts of image quality.

The first paper, by I. Overington, is a review of much of his work over the past few years, which concerns the development of a visual model. In addition to covering his own work, the article also presents an excellent overview of research into the area of vision and the attributes of imagery important to perception.

The next two articles deal with subjective ratings of imagery. H. Snyder, J. Burke, et al. discuss their work on preparation of a data base and imagery for subjective studies. They also report on subjective ratings of various image blurrings. The paper by R. Arguello, H. Kessler, and H. Sellner discusses subjective ratings of images produced from synthesized MTF shapes.

Turning to imaging systems and methods, we have $\mathrm{H}$. Edgerton's paper on techniques of shadow imaging, including method, application, and error sources. This paper is followed by $\mathrm{H}$. Pollehn's discussion of current methods of evaluation and specification of image intensifiers.

P. Peters presents a paper on his work to model an electro-optical imaging system from end-to-end in order to simulate image degradations arising from each element of the total chain.

The paper by V. Kumar, D. Casasent, and H. Murakami represents an entirely different quality problem. Pattern recognition work depends on the strength of the recognition. Improvement in interpretation results in improved image quality. Perhaps other disciplines will make similar quality improvements simply through data processing rather than system modification.

The last article concerns an especially interesting area, imaging with nonvisible wavelengths. $\mathrm{R}$. Mitchel and $\mathrm{S}$. Marder review the field of synthetic aperture radar imaging, outlining the important considerations, including system parameters and image degradations.

This special issue is a compilation of approaches to image quality. We hope that it will serve as a catalyst to interaction between different disciplines. 